\title{
Human attribute concepts: Relative ubiquity across twelve mutually isolated languages
}

\author{
Saucier, Gerard ; Thalmayer, Amber Gayle ; Bel-Bahar, Tarik S
}

\begin{abstract}
It has been unclear which human-attribute concepts are most universal across languages. To identify common-denominator concepts, we used dictionaries for twelve mutually isolated languages Maasai, Supyire Senoufo, Khoekhoe, Afar, Mara Chin, Hmong, Wik-Mungkan, Enga, Fijian, Inuktitut, Hopi, and Kuna - representing diverse cultural characteristics and language families, from multiple continents. A composite list of every person-descriptive term in each lexicon was closely examined to determine the content (in terms of English translation) most ubiquitous across languages. Study 1 identified 28 single-word concepts used to describe persons in all 12 languages, as well as 41 additional terms found in 11 of 12 . Results indicated that attribute concepts related to morality and competence appear to be as cross-culturally ubiquitous as basic-emotion concepts. Formulations of universal-attribute concepts from Osgood and Wierzbicka were well-supported. Study 2 compared lexically based personality models on the relative ubiquity of key associated terms, finding that one-and two-dimensional models draw on markedly more ubiquitous terms than do five-or six-factor models. We suggest that ubiquitous attributes reflect common cultural as well as common biological processes.
\end{abstract}

DOI: https://doi.org/10.1037/a0036492

Posted at the Zurich Open Repository and Archive, University of Zurich

ZORA URL: https://doi.org/10.5167/uzh-210055

Journal Article

Accepted Version

Originally published at:

Saucier, Gerard; Thalmayer, Amber Gayle; Bel-Bahar, Tarik S (2014). Human attribute concepts: Relative ubiquity across twelve mutually isolated languages. Journal of Personality and Social Psychology, 107(1):199-216.

DOI: https://doi.org/10.1037/a0036492 


\section{Human Attribute Concepts:}

Relative Ubiquity Across Twelve Mutually Isolated Languages

Gerard Saucier, Amber Gayle Thalmayer, and Tarik S. Bel-Bahar University of Oregon

\section{Acknowledgement}

Thanks to David Evans, Jennifer Miner, and Miwa Ogo, who provided substantial research assistance. Tarik S. Bel-Bahar is now at the Department of Psychology and Neuroscience, Duke University. We thank Lisa DiBlas and Michael C. Ashton for providing lexical data and/or results utilized in a part of Study 2. Correspondence may be addressed to: Gerard Saucier, Department of Psychology, 1227 University of Oregon, Eugene, OR 97403, USA (E-mail: gsaucier@uoregon.edu) 


\begin{abstract}
It has been unclear which human-attribute concepts are most universal across languages. To identify common-denominator concepts, we used dictionaries for twelve mutually isolated languages -- Maasai, Supyire Senoufo, Khoekhoe, Afar, Mara Chin, Hmong, Wik-Mungkan, Enga, Fijian, Inuktitut, Hopi, and Kuna -- representing diverse cultural characteristics and language families, from multiple continents. A composite list of every person-descriptive term in each lexicon was closely examined to determine the content (in terms of English translation) most ubiquitous across languages. Study 1 identified 28 single-word concepts used to describe persons in all 12 languages, as well as 41 additional terms found in 11 of 12 . Results indicated that attribute concepts related to morality and competence appear to be as cross-culturally ubiquitous as basic-emotion concepts. Formulations of universal-attribute concepts from Osgood and Wierzbicka were well-supported. Study 2 compared lexically based personality models on the relative ubiquity of key associated terms, finding that one- and two-dimensional models draw on markedly more ubiquitous terms than do five- or six-factor models. We suggest that ubiquitous attributes reflect common cultural as well as common biological processes.
\end{abstract}

Keywords: personality, emotion, language, morality, competence, Big Five, Big Six 
Psychology is the study of human minds and behaviors, not just humans speaking one language or in one kind of cultural setting. Accordingly, psychologists should be interested in the degree to which the concepts they use are actually widely applicable. Some concepts may be primarily applicable to only one kind of population, whereas others may have a wider currency. Likely advantages of using widely referenced concepts would be easier translatability, better cross-cultural applicability, and perhaps greater social importance.

The current studies aim to distinguish the most ubiquitous (widely referenced, potentially universal) human-attribute concepts from those of more limited distribution. Ubiquitous concepts are potentially universal, where universal means "characteristic of all members of a class, without limit or exception" (Allik, Realo, \& McCrae, 2013, p. 61). Here, "human attribute" means a characteristic or quality -- transient or enduring, physical or psychological -- ascribable to a human person, and not delimited to a part of a person (like the voice or the eyes). To distinguish ubiquitous attribute concepts, the best data would be that with the highest degree of

variance in attribute concepts. A diverse set of mutually isolated languages would be one place to find such high-variance data.

Which Concepts Are Universal?

Words are units of language, while concepts are units of meaning. Many of the mental representations we think of as concepts are represented by terms in language, although arguably each separable meaning of such a term is a separate concept. Thus, a term does not always correspond neatly to one concept. Many attribute terms are polysemous and represent multiple concepts, only one of which might be a human attribute. For example, the most common meanings for English terms like deep and kind are not human attributes, but for each of these the attribute-concept is singular in meaning. Polysemous in a different way are terms like curious and good, each of which has two major competing meanings -- morally good versus competent at something; strange versus inquisitive. Most often, however, a given human-attribute-descriptive term roughly corresponds to one concept. Accordingly, in what follows, we generally equate 'term' with 'concept,' but do not apply this equivalence rigidly. 
Which human-attribute concepts are universal, showing the least cross-cultural variation? Cross-cultural common denominators of many sorts have been proposed (summaries are provided by Murdock, 1945; Brown 1991). For example, there has been interest in linguistic universals (Greenberg, 1966, 1975), and Wierzbicka (1996) proposed a specific set of semantic universals that included attribute concepts like good, bad, big, and small. A better understanding of possible psychological universals is important for studies of culture and personality (Church, 2008; Heine \& Buchtel, 2009) and psychology in general (Cheung, van de Vijver \& Leong, 2011; Norenzayan \& Heine, 2005).

In psychology, universals have been proposed for affective meaning: Osgood and colleagues determined that in judgments about any objects humans tend to use dimensions of evaluation, potency, and activity (Osgood, 1962; Osgood, May, \& Miron, 1975). Following formulations by philosophers (Descartes, Hobbes, Spinoza) of "building block" emotion categories (Solomon, 2002), proposals have been made for a set of basic human emotions (Ekman, Sorenson, \& Friesen, 1969; Hupka, Lenton, \& Hutchison, 1999; Izard, 1992; Plutchik 1980; Tomkins, 1970). The lists of basic emotions vary, but proposals do tend to converge in identifying as basic categories fear, anger, joy/happiness, sadness or distress, disgust, surprise, and perhaps also interest and contempt. However, previous work has not adequately addressed either the potential universality of concepts of personality or of the broader class of human attribute-concepts of which personality is a subset. The current report fills that void.

\section{$\underline{\text { Universal Personality-Attribute Concepts? }}$}

The term 'personality' refers to relatively stable patterns of behavior, affect, and thinking. In studies of personality lexicons, there is controversy over whether to select terms restrictively or broadly (Saucier \& Goldberg, 2001); should we define personality narrowly as intrinsic traits, or broadly as any attribute representing a pattern of behavior, affect, or thinking, from either a self or an external-evaluator standpoint? Lexical studies of personality have used narrow selections (e.g., Lee \& Ashton, 2008) or broad selections (e.g., Saucier, 2009a). For purposes of identifying the most universal concepts of personality it is helpful to begin with a maximally 
broad strategy, retaining terms representing evaluations of a person, the person's status or effects on others, temporary states, or physical attributes. Thereby, we can directly compare the ubiquity of concepts for personality and concepts for emotion, physique, and other possible categories. To the extent the inclusiveness of the concept of personality is ambiguous, this approach avoids any unnecessary commitment to a particular degree of narrowness versus inclusiveness.

The present project was primarily descriptive in nature. Its main goal was to catalog human-attribute concepts represented in single words within a maximal range of languages. However, once that corpus was composed, we were able to consider a full range of potential hypotheses (based strictly on previously identified models involving other investigators) about which human attribute concepts would prove most ubiquitous. Some (Osgood, Wierzbicka, basic emotions) have already been mentioned. Two more pertain more directly to personality.

It has been proposed (since Goldberg, 1981) that the Big Five personality dimensions Extraversion, Agreeableness, Conscientiousness, Emotional Stability, and Intellect (sometimes Openness ) - might be universals, derivable from the study of any human language. The fivefactor approach has become the dominant model in personality research, but claims of its universality are based on relatively loose, lenient criteria, such as whether a measure of the five factors holds much of its hypothesized structure after being translated into other languages. Examinations of its universality by more stringent criteria are warranted. One more stringent approach is to conduct indigenous lexical studies in a diverse range of languages; these studies reveal limitations to the universality of the five dimensions (Saucier, 2009b). In the absence of indigenous lexical studies in every language, it would be useful to examine whether attribute terms with Big Five factor content are found in all languages, since appearance of a dimension presupposes that the language has words representing it.

Social psychologists have proposed that there are two important and differentiable types of content in perception and judgment of self and others. One is consensually labelled competence, with the other labelled either as morality (Wojciszke, 2005a, 2005b) or warmth (Fiske et al., 2006; Judd et al., 2005). Morality and warmth are related: Fiske et al. (2006) refer to the warmth 
dimension as morality or moral-social, and Leach, Ellemers, and Barreto (2007) identified warmth/sociability and morality as two components within the Fiske et al. construct of warmth (cf. Rosenberg et al., 1968). Fiske et al. (2006) have claimed universality for these dimensions. Inferring Universals

All known human languages include numerous terms referring to attributes of personality and other human propensities (Dixon, 1982). But languages differ in the array of specific personality attributes efficiently represented in single words in the language (Dixon, 1982).

How would one infer universality? A universal would be found in all cultures. Strictly speaking, universality could be inferred only if cultures were studied, which is impossible. We infer universality based on observed ubiquity - empirical occurrence in all members of a representative subset of all cultures. Observed ubiquity means a high probability of universality.

One might contrast an empirical approach with a post-modernist view in which identification of universals is neither desirable nor possible (Fish, 1999; cf., Raybeck \& Herrmann, 1996). This view puts a brake, usefully, on premature identification of universals. Post-modernism implies that observed ubiquity - seeming universality - will never translate into real universality. In order to test such a view with respect to attribute concepts, we would need a candidate set of concepts that have been initially observed to be ubiquitous. The task for this study was deriving an optimal, empirically based candidate-set of ubiquitous concepts.

Identification of ubiquitous human-attribute concepts will prove useful in several ways. First, ubiquitous concepts point toward important "necessary but not sufficient" components for basic psychological science. Any good model of psychological dispositions should take account of those attribute-concepts that are most universally distributed across languages. These can serve as a "ground" for understanding (as "figure") those attributes with the most variation across cultures. Second, these important components - by virtue of their ubiquity - may point toward important causal or contextual variables (e.g., biological factors, or socio-cultural factors operating similarly across populations). Third, identifying ubiquitous human-attribute concepts can help intercultural communication by spotlighting concepts with the widest currency, 
logically constituting the set of concepts most easy to translate and utilize across populations, for use in an increasingly globalized psychological science community.

We examined the human-attribute content found in 12 languages that are not only mutually isolated, but also diverse in geographical and cultural context. This combination - mutual isolation and high diversity - makes for a strong test of cross-cultural ubiquity, because in such a set of languages, one cannot easily attribute similarities to mutual influence or to the effects of third variables. This study compared the sets of concepts arising spontaneously within each of these 12 languages, to find common denominators with respect to descriptions of human attributes. Our focus was not on mere translatability. With enough effort and allowing lengthy phrasing, one might roughly translate most concepts from one language to another (an etic approach). But we focus here on concepts that are potentially indigenous (emic) to all languages.

Finally, an important interpretational point: We do not assume that populations speaking certain languages are incapable of understanding a particular concept if they happen to have no word for it. There is no word in English for the German schadenfreude (taking pleasure in the misfortune others), but that does not mean English-speakers are incapable of grasping the concept. The lack of word does however imply that this particular language-community has not in the past tended to emphasize the distinction: We assume that whether a language does or does not have a word says more about habit than about ability.

\section{Study 1}

\section{Method}

Assume there were a universal human-attribute concept: What would follow? The lexicon of any language (e.g., that for Arapaho, or that for Zulu) would have a term referencing it, or at least some variation on its basic theme. And therefore, a dictionary created to encapsulate that lexicon would contain one or more such terms. Moreover, if a set of dictionaries rendered translations of the terms in their lexicons into some common language (e.g., English), then a single term for that universal concept from that common language would be used as a translation of at least one such term from any of a wide array of languages. 
Accordingly, one might identify potential universals by attending to a suitably wide array of languages, each having a dictionary that provides a translation of that language's terms into a single common language (e.g., English). Although precise translations of that original language's (e.g., Zulu's) concepts may be affected by the second-language (e.g., English) word preferences of the particular translator, the character of the body of terms in the dictionary is driven by the original language. Thus, the strategy of relying on a lexicon presenting the original language's corpus of concepts is superior to the reverse approach, one examining the lexicon of a single language trying to find equivalents in a maximal number of other languages (e.g., a list of English words giving for each its closest Zulu equivalent). The terms included in such reverse lexicons will tend to represent the conceptual preoccupations of the imported, common language (e.g., English) and not that of the local language.

Finding a single, workable, common language is not difficult. Although a number of languages (e.g., French, Spanish, Russian) have been used to communicate the meanings of one language into terms understandable in another, English is currently the most widely used for this purpose; indeed it is unlikely, at this point in time, that a study like the present one could be done with any other language as the common denominator. Importantly, the translations used here were produced by experts unaffiliated with our research team, for purposes having nothing to do with our study. Thus, these translations cannot reflect biases of the present investigators.

What is a "suitably wide array of languages"? Ideally, languages (a) from a variety of the world's language families, used by groups (b) in widely varying geographical regions that (c) reflect a wide range of cultural features. We could not rely on existing 'trait taxonomies' to generate a suitably wide array, as these draw preponderantly from Indo-European languages spoken by European-origin populations and from complex societies having relatively similar forms of social organization. One must turn to traditional societies, because these have greater degrees of diversity and mutual isolation. To select languages based on these criteria we used scientific classifications of language families, a world map, and an 'atlas' (Murdock, 1967) of how specific ethnographic features are distributed across 862 traditional human cultures. 
To select a tractable number of languages (12) that were as diversified as possible, we began by selecting a few languages (Khoekhoe, Supyire Senoufo, Maa, Fijian, Hopi) for which we early on (through the recommendation of linguists) identified highly detailed dictionaries that provided English glosses. Noting the ethnographic features associated with these initial languages, we then identified languages from other world-regions whose inclusion would best complement them and maximize the diversity of the total set of 12 , making the proportional tendencies of the 12 societies match the proportional tendencies of the world cultures as surveyed in the ethnological literature. Table 1 lists the 12 languages selected, including the dictionary used and the geographical provenance of the language. Information on language selection and the traditional cultural, ethnographic features of the societies associated with these languages (from Murdock, 1967) is available in the Appendix. Murdock (1967) gathered in a single volume the 21 installments of an ethnographic atlas published in an anthropology journal across several years. Features listed in the Appendix pertain to the traditional (not necessarily the contemporary) culture of the group as documented in ethnological sources through the mid-20 ${ }^{\text {th }}$ century, and are selected to emphasize what makes each group most distinct from the other 11. The table does not list all ethnographic features: Many of these cultures traditionally had other characteristics that are fairly widespread across world cultures, and thus poor differentiators, such as: gift-exchange or bride-price associated with marriage, extended or nuclear families, patrilocal residence, patrilineal kin groups, agamous or clan organization, communities with more than 50 persons, bovine animals, and an absence of castes or slavery.

The Appendix also includes information relevant to placing the societies on Service's (1962) continuum of social organization, according to which non-state societies are classified as having egalitarian band versus more complex hierarchical chiefdom social organization (with what Service labels "tribe" organization falling between band and chiefdom). This is intended as a descriptive and not evaluative characterization; one might see egalitarianism and complexity as both being advantageous; through history human societies have frequently traded off one for the other (although contemporary social democracies attempt to achieve both simultaneously). 
The best available dictionary for each language was selected, provided it rendered the meaning of each entry into English (there was usually only one good choice in each case). Each dictionary contained at least 6,000 entries. Some comparisons are relevant: The most frequentlyused 6,000 words in English account for some 90 percent of words in English texts (Francis \& Kucera, 1982), and nearly 100 percent of those used in informal speech (Schonell et al., 1956). Two of us (the first and third authors) went through each dictionary term by term, marking and extracting any term that might conceivably be used to describe a human (not merely a body part of a human, as in "green eyes"). Having two independent extractions ensured that no relevant terms were omitted; a term was retained if extracted by either of the two. In total, 16,857 original-language terms referring to human attributes were extracted from the 12 dictionaries.

As Table 1 indicates, languages varied in number of original-language terms extracted. This variation clearly proceeded from two sources: Dictionaries differed in their length and detail, the longer dictionaries having more terms to extract (across the 12 languages, the number of entries predicted the number of attribute terms with a correlation of .69), and they also differed in the degree to which related words sharing a root were split into multiple entries or condensed into one or a few entries. Sometimes two different orthographies (spellings) were presented as two entries for essentially the same term: The 1,816 terms extracted from Inuktitut included many terms that were small orthographic variations on another term. Sometimes a single entry generated multiple distinguishable terms: The 3,456 terms extracted from Afar were from 3,378 separate dictionary entries: some entries listed multiple terms. Thus, one cannot necessarily infer that some languages have substantially more terms for describing human attributes. A shorter list of terms would arise from a dictionary that focused on frequently used terms, or that did not treat each variation on a root as a separate term, as well as from any undersampling of attribute-terms.

The 12 languages varied considerably in the percentage of total entries that were extracted as human-attribute terms. Ten had from 9 to 23 percent extracted as attribute-terms. Kuna had just five percent, likely evidence of systematic undersampling of human-attribute terms by the 
dictionary author. Afar had 36 percent, although this language had 115 cases where there were separate words/entries for an attribute according to whether a male or a female possessed it, which would tend to inflate the number of extractable attribute-terms.

The 12 languages vary considerably in the number of native speakers, from over 1 million for Afar and Maa and 600,000 for Hmong at the high end, down to about 14,000 for Eastern Canadian Inuktitut, 5200 for Hopi, and 800 for Wik-mungkan. There was no significant correlation between number of speakers and number of either entries or attribute-terms extracted.

Moreover, as examination of Table 1 would suggest, there was no significant correlation between a rank-ordering of the societies on the band versus chiefdom continuum of social organization (Service, 1971), and any of three lexical quanta: (a) the total number of terms in the dictionary, (b) the number of human-attribute terms, and (c) the ratio of (b) to (a). There is no evidence here that hunting/gathering peoples distinguish personality and individual differences any less (or more) than herding or farming peoples. At least among traditional societies, there is no evidence of social complexity affecting the size of the lexicon for attributes on which people differ. These attributes seem to be widely referenced everywhere, in all kinds of human societies, with no evident effects of "stages of development" on the relative abundance of attribute terms.

In this study we assumed that a ubiquitous concept will be represented by a single, ubiquitous term. What counts as a ubiquitous term? By the simplest approach, ubiquity occurred wherever the same exact English term, or a variant on its same root, appeared as a gloss in all 12 languages. As an example of variants on the same root that were counted as the same term in our approach, consider "fool," "foolish," "foolishness," and any verb glossed as "to be foolish." Thus our understanding of a "term" is a word-root that might appear in a variety of word-forms. We identified the most ubiquitous terms by careful checks on several hundred terms that appeared most frequently in the corpus when all English entries were split into their constituent words (in most dictionaries, it was common to find several English glosses for each native-language term).

We then compared the resulting list of ubiquitous terms to expectations generated by prior theories, which are as follows. According to post-modernistic cultural relativism there would be 
no ubiquitous terms at all. According to Osgood's theory, there would be ubiquitous evaluative terms (e.g., good-bad) as well as terms indicating potency (e.g., strong-weak) and activity (e.g., fast-slow). According to the "semantic primitives" theory of Wierzbicka, there would be ubiquitous terms for good-bad and big-small. According to proposals in theories of basic emotions, there would be terms for anger, fear, sadness or distress, joy or happiness, surprise, and disgust, and perhaps contempt and interest. According to a Big Five or five-factor personality theory (Goldberg, 1993; McCrae \& Costa, 1997), there would be ubiquitous terms representative of Agreeableness, Conscientiousness, Emotional Stability (vs. Neuroticism), Extraversion, and Intellect/Openness dimensions. A less ambitious proposal is that there would be ubiquitous terms representing both morality (or warmth) and competence, as proposed by social psychologists. All of these theories might be interpreted in either a strict or lenient version: strictly, that the only ubiquitous concepts found would be those identified by the theory, or leniently, that among the ubiquitous concepts found would be those that the theory identifies.

\section{Results}

The terms in Table 2 are English terms that linguists relied on most often in order to describe the meaning of frequently used terms in languages they were documenting. The leftmost column of Table 2 presents that set of concepts referring to human attributes found in all 12 languages, based on English translations provided by the dictionary-makers. Concepts are grouped into various classes of attributes, including evaluation, size, potency and debility, age, emotion, and, for behavioral dispositions, relevance to morality and competence. It seems safe to say that at least half the concepts in Table 2 represent attributes studied in psychology.

In addition to the 24 concepts clearly descriptive of human attributes, there are four concepts (cold, hot, hard, dirty) for which there is some ambiguity as to whether their variants in every language are used to describe humans; these are terms most commonly used to describe non-human objects. We do not claim that they are necessarily human-attribute universals, but despite their marginality we include them, for the sake of complete documentation. 
In order for a concept truly present and salient in all 12 languages to appear on our list, there was no margin for error on the part of dictionary-authors. However, in creating a dictionary it is inevitable that a few relatively commonly used terms in the original language will remain unsampled due to the particular sources used at a particular time and place. A looser criterion would tolerate an occasional error of omission in the dictionaries, by only requiring that a concept appear in 11 of 12 languages. The center column of Table 2 provides 29 further concepts meeting this looser criterion, of which about half represent attributes studied by psychologists. It contains also 12 further (marginal) concepts for which there is some ambiguity whether variants in every language are used to describe humans. Notably, the concepts in the center column include an additional grouping of motivational states (hunger, thirst, pain, pleasure) that should obviously be human universals; finding these concepts here tends to support allowing a slight margin of error and using evidence from 11 of 12 languages as a criterion for ubiquity.

We noted earlier our suspicion that the Kuna dictionary had undersampled human-attribute terms, based on a low proportion of total entries. Of the 41 concepts appearing in 11 of 12 languages, nearly half (20) were missing only in Kuna. The more lenient criterion of "at least 11 " rather than "all 12" languages helps to correct for any undersampling in Kuna.

One could divide the concepts represented in languages into three groups: those found in all cultural settings, those found in only some settings, and those unique to one setting only (as in Georgas \& Mylonas, 2006). Results here indicate those found in all languages are a small group. Interestingly, in Table 2 a large proportion - nearly half -- of the ubiquitous attribute concepts are in marked semantic oppositions to other concepts. Particularly salient are 15 such pairs: Good-Bad (or the partly synonymous Good-Evil), Large-Small (or Big-Little), StrongWeak, Sick-Well, Old-Young, Cold-Hot, Dirty-Clean, Soft-Hard, Short-Tall, Quick-Slow, RichPoor, Right-Wrong, Straight-Crooked, Pain-Pleasure, and Wise-Foolish. For these 15 pairs, both ends of the contrast appeared in at least 11 of the 12 languages examined. This is consistent with a tendency for human languages to organize attributes into antonymous pairs, and with such formulations as Harter's (2001) developmental theory of trait knowledge and Kelly's (1995) 
cognitive theory of personality. On the other hand, many concepts in Table 2 (e.g., ashamed, jealous) do not have specific opposites other than rarely-used negation terms (i.e., unashamed, unjealous) in English. Thus, one should not assume that concepts are necessarily less important if they are without a widely-found semantic opposite (cf., Rozin, Berman \& Royzman, 2010).

Results clearly support several of the theoretical conceptions outlined earlier. Wierzbicka's semantic primitives of good-bad and big-small is strongly supported by the literal appearance of these terms in Table 2. However, these may not be the only universal human-attribute concepts.

Osgood's conception of a dominant Evaluation dimension in judgments about objects of any kind is strongly supported by the appearance of many ubiquitous evaluative concepts (good, bad, beautiful in all 12 languages, evil, foolish, handsome, important, useless in 11). Osgood's additional Potency dimension is well-supported: A strong-weak distinction is evident in all 12 languages. As for Activity, concepts of quick and slow were found in 11 of 12 . But ubiquitous human-attribute concepts do not seem strictly limited to Evaluation, Potency, and Activity.

While basic-emotions theorists lack perfect agreement on what the most basic categories of emotion are, Table 2 includes labels for some of the most frequently mentioned: fear (afraid), anger, surprise, joy (happy), and distress (troubled). Basic emotion concepts that are frequently mentioned in the research literature but for which labels do not appear in the tables include disgust, sadness, contempt, and interest. Sadness appeared in only 10 of these 12 languages (missing in Kuna and Hmong), and the other three concepts in less than 10. However, the ubiquitous concept "dirty" appears to be often (in these languages) synonymous with "disgusting", the concepts "stupid" and "foolish" imply contempt on the part of the observer who is making the judgment, and "love" might be taken to imply interest. Overall, the results provide some support for conceptions of basic emotion categories.

Even so, results indicate that ubiquitous psychological attributes are not limited to the domain of emotions. The concepts disobedient, stupid, and jealous were found in all 12 languages, and the concepts clumsy, humble, wise, unable, gossip, and love were found in 11. Of these ten terms, as the groupings in Table 2 show, five can be linked to morality, as attributes 
related to care, fairness, and loyalty, which constitute diverse "moral foundations" (Haidt, 2007). Four others can be linked to competence. We note however that this set of ubiquitous behavioral descriptors does not lend obvious support to current conceptions of basic personality-trait dimensions (e.g., the Big Five) that go beyond moral character and competence.

Searching for further behavioral tendencies that were frequently if not ubiquitously represented, we identified concepts present in single words in 10 of the 12 languages. The most personality-related of these concepts were bold, brave, clever, intelligent, skilled, careful, forgetful, wild, busy, sad, worried, stubborn, liar, pity, trustworthy, and wicked. These 16 terms arguably involve fewer than 16 content classes. Every one of the 12 languages had a word for either bold or brave, and these are synonymous (involving courage). ${ }^{1}$ Every language had a word for either careful or forgetful, and for either wicked or trustworthy (or, for either wicked or liar); these are not particularly close synonyms, but they have enough overlap of meaning to imply a possible broader content class (e.g., Conscientiousness or Honesty). If there is any support in these data for a relatively complex theory of multiple personality dimensions like the Big Five, it would be at this secondary level, where a content-class might be inferred to be universal even if none of the specific concepts within the class are.

\section{Discussion}

This study applied relatively stringent criteria for the ubiquity of a concept: That it be represented in at least 11 of 12 mutually unrelated languages by one or more single-word concepts, concepts used frequently enough to make their way into a dictionary of at least 4200 words, or a minimum of about 300 human-attribute concepts. Our use of relatively stringent criteria enables us to separate likely "truly ubiquitous" concepts that from those that may merely be potentially universally useable via careful translation across languages.

Ubiquitous attribute concepts appear to be islands in a sea of intercultural variability, with few clear 'universals. Proportionally few attribute concepts appeared to generalize across these languages. However, many of these "islands" appear well-mapped by existing theories drawn from the fields of emotion, linguistics, and social psychology. Results supported several of these 
theories simultaneously, including those associated with semantic primitives (Wierzbicka, 1996), universals of affective meaning (Osgood, 1962), morality and competence (Leach et al., 2007; Wojciszke, 2005a, 2005b), and some basic emotion categories proposed by various psychological theorists (Ekman, Sorenson, \& Friesen, 1969; Hupka, Lenton, \& Hutchison, 1999; Izard, 1992; Plutchik 1980; Tomkins, 1970). Results did not favor one of these theories to the exclusion of the others. These theories all go against a strict post-modernist cultural relativism. But strict cultural relativism - positing that no "anchor points" or universal units in cultural comparisons can be found -- might still be vindicated if every one of the apparently ubiquitous human-attribute concepts identified in Table 2 were found to be missing in one or more human languages, or in at least 5\% of them (after Brown, 1991). Future studies might resolve the matter.

The potential universality of certain semantic primitives, of affective-meaning dimensions, and of basic emotion concepts is relatively unsurprising; what may be more surprising is that a single study would support all three of these approaches simultaneously.

Emotions are regarded as short-lived, attention-capturing phenomena that momentarily strongly impact behavior, facial expression, physiology, and subjective experience. It bears noting that neither love nor jealousy is consensually considered to be an emotion by psychologists who study emotions. Love involves attraction, admiration, concern, and devotion that are often sustained over long periods of time, whereas jealousy is likewise often sustained, and involves apprehension, suspicion, intolerance, and vigilance in addition to emotional distress. The finding that love and jealousy concepts are relatively ubiquitous is in line with proposals of evolutionary psychology (Buss, 2008; Tooby \& Cosmides, 2000), which are that jealousy and love (not to mention beauty/handsomeness) evolved to address adaptive problems associated with reproduction and are species-typical, that is, universal aspects of human nature.

One key new finding from this study is the potential universality of a variety of concepts descriptive of behavioral tendencies. These have some coherence if one thinks in terms of a few foundational constructs. There is a distinct emphasis among the most ubiquitous concepts on broad-level evaluations, with respect to the utility of a person in broad domains like morality, 
competence (skills, potency, debility), and attractiveness. Fiske et al. (2006) have specifically claimed universality for dimensions of competence and warmth/morality, which was bold since their data emphasized only Western college students. However, findings here are generally supportive of such claims, though more for the universality of morality than the warmth/sociability component (cf. Leach et al. 2007; Wojciszke, 2005a, 2005b).

A second new indication is that the content of these potential universals seems to conform poorly to the outlines of recent models of the most important personality dimensions, such as the "Big Five." Why this anomaly? These recent models may be derived from too narrow an evidentiary basis with respect to the diversity of human cultural contexts. These recent models do not place at their center those personality-attribute concepts that seem most likely to have arisen spontaneously in the widest range of cultural settings; that is, they are not closely anchored to the "islands in a sea of interculturally variable concepts" mentioned earlier. As a result, a model like the Big Five seems more vulnerable to a cultural-relativism critique than are the other theories examined here. ${ }^{2}$

Is the Big Five ubiquitous, by a stringent criterion? Among the 69 terms in Table 2 only four -- Cold, Angry, Fearful, and Jealous -- are identified as Big Five markers anywhere within Goldberg's classic article on adjectives for the Big Five (Goldberg, 1992), and these four terms represent only two of the Big Five dimensions (Agreeableness and Emotional Stability). There is no indication that recently proposed six-factor models (Lee \& Ashton, 2008; Saucier, 2009a) fare better in respect to terms in Table 2.

A better case can be made that the attribute concepts in Table 2 provide support to two factors each broader than the Big Five. These may appear as higher-order factors from analyzing Big Five intercorrelations (DeYoung, 2006), or in analyses that extract only two factors at the “first-order" level (Saucier, 2009b). These two factors have some support from cross-cultural studies (Cuddy et al., 2009) and the neuroscience of personality (De Young, 2010).

It might be objected that this pattern of results, discouraging with respect to personality and person-perception models having more than two dimensions, arises from the stringent criterion 
used in the study: That a concept can only be regarded as ubiquitous if it appears in the form of a given English term (i.e., word-root) in at least 11 of 12 unrelated languages. A less stringent criterion would consider a concept ubiquitous if any of several related terms - a synonym cluster -- appear in all the languages. There is not a consensual scheme for clustering human-attribute terms, even just in English, but if one were presented it would deserve attention in these data.

But fortunately, we do have (lexical) studies of multiple languages with respect to personality factors, which in many cases enable us to derive conceptions defining the core of each factor based on recurrence across at least some set of languages. In a second round of analyses using a less stringent criterion (i.e., Study 2), we examined the relative ubiquity across the same 12 languages of clusters of human-attribute concepts, where each cluster is identified with a recurrent dimension in the description and evaluation of human personality.

\section{Study 2}

Reviews (e.g., Saucier, 2009b; Saucier \& Goldberg, 2001) indicate that previous lexical studies of personality - to varying degrees, including other human-attribute terms - have provided evidence to support more than one structural model. These structural models vary principally in how many dimensions they contain. Structures with one, two, three, five, and six dimensions have received the most attention.

The most parsimonious such structure has just one dimension, generally identified with global evaluation. There has been, to date, no published study of the terms recurrently associated with this one dimension across languages. However, Osgood and colleagues (Osgood, May, \& Miron, 1975, Table 4:18) recommended various scales for measuring pancultural evaluation in a short-form semantic differential, which can be adapted for this use. As Saucier (2008, p. 33) noted, eight terms (in four pairs) were particularly useful cross-culturally (recommended for at least three of the 21 languages): good-bad, pleasant-unpleasant, beautiful-ugly, and nice-awful. This provides a short set of seven word-roots (unpleasant having a redundant root with pleasant). For a longer set we selected those twelve additional terms, from among those 
suggested by Osgood et al. for at least two languages, most clearly applicable to description of humans. Table 3 presents both the shorter and longer list of Evaluation terms.

At the two-factor level, social self-regulation and dynamism are found recurrently across many languages. Saucier et al. (in press) identified 10 terms (translated into English) most recurrently associated with social self-regulation, and likewise seven terms highly associated with dynamism. A more relaxed criterion (4 of 9 languages) would include 16 additional Big Two marker terms, yielding a longer set of synonym clusters for each of these two factors. Big Two markers from Saucier et al. (in press) are found in Table 3. ${ }^{3}$

Despite recent interest in a replicable set of three lexical factors, no study has specified the terms most recurrently associated with it. Clearly, this Big Three does not closely resemble the E-P-A dimensions of Osgood discussed earlier in this paper. Because the labels heretofore applied to the Big Three are the same as for three of the Big Five (Extraversion, Agreeableness, and Conscientiousness), they are often assumed to be identical to three Big Five dimensions, although it is likely that some are somewhat broader than the Big Five dimension with the same name. A similar situation pertains to any possible 'Big Four': No study has specified which terms are most recurrently associated with four-factor solutions in lexical studies. It is generally assumed that any 'Big Four' would be essentially the Big Five minus the so-called "fifth factor" (Intellect, Openness), thus converging with the four factors found in personality psychopathology (Calabrese, Rudick, Simms, \& Clark, 2012).

Nonetheless, one might surmise the kind of descriptors that would be associated with three- and four-factor lexical models by examining previous studies. One simple approach is to assume that terms associated with Big Five factors (Extraversion, Agreeableness, and Conscientiousness for the Big Three, with the addition of Emotional Stability for the 'Big Four') are adequate representatives. A limitation is that this approach treats the Big Three and Four only as truncations of the Big Five, not containing any factors broader than the Big Five. As an alternative, one might consult the analyses of 14 trait taxonomies by De Raad et al. (2010). There, Table 7 indicates that four-factor solutions from Italian (Trieste) and English (Canadian) 
studies (DiBlas \& Forzi, 1998; Lee \& Ashton, 2008) have the highest average congruency with four-factor solutions derived from other languages. Three-factor solutions from these two studies likewise are among the three with the highest average congruency with three-factor solutions from other languages. ${ }^{4}$ Accordingly, one might treat these two studies as revealing a prototypical Big Three and Four. Following this rationale, we sought those descriptors that (using English translations for the Italian terms) the most salient on factors derived from both languages, with the aim of yielding a set of terms for each factor of roughly equal magnitude as for the other models tested here. For each pole of each factor in three- and four-factor solutions, the 26 terms with the highest loadings were identified, and these were retained as critical terms if found on the same factor in both languages. Table 3 indicates the terms meeting this criterion.

Turning to the Big Five, conceptions defining the core of each factor can be derived from the study of De Raad, Perugini, Hrebickova, and Szarota (1998). These authors compared fivefactor structures in seven languages - Dutch, German, Czech, Polish, Italian, Hungarian, and English. Table 2 of their paper presents 16 adjectives for each of five factors in each language eight for each pole of each bipolar dimension. Here, Table 3 identifies the 45 terms identified as salient terms for the same factor in at least three of the seven languages. This seven-language composite contains the most cross-culturally recurrent lexical Big Five identified to date.

Another representation of the Big Five in adjective form has been 100 adjectives selected by Goldberg (1992; later abbreviated by Saucier, 1994). Although this representation arises entirely out of studies with the English lexicon, it has been more widely used than any other adjective-based measure, and it served as basis for "IPIP" measures of the Big Five (Goldberg et al., 2006). Table 3 includes those 85 of Goldberg's adjectives that have non-redundant wordroots. Note that in Goldberg's representation Agreeableness emphasizes warmth and sympathy, whereas the cross-language version emphasizes peacefulness versus aggressive dominance.

Representations of a six-factor structure have been derived from lexical studies using relatively narrow selections of personality variables (Ashton et al., 2004) and from those using relatively broad selections (Saucier, 2009a); the two representations are highly related but non- 
equivalent. Table 3 includes adjectives with non-redundant word-roots representing each of these structures. The HEXACO structure is based on narrower variable selections; these are adjectives identified as salient on each factor in at least three of the eight studies analyzed by Ashton et al. (2004), as shown by Saucier (2009a, Table 1). The 'Big Six' adjectives in Appendix B are those identified as salient in at least two of the eight studies considered by Saucier (2009a, Table 4).

The sets of descriptors used (see Table 3) assign roughly equal numbers of terms-per-factor for each model, once one aggregates across the two variants. The variant Evaluation measures average 13 terms per factor, with a corresponding average of $12.5,12.7,13.25,13$, and 12.4 respectively for Big Two, Big Three, Big Four, Big Five, and Big Six factor sets. Thus, overall results should not be biased by the number of terms representing each factor.

\section{Method}

The same corpus of 16,857 human-attribute-term entries extracted from the 12 languages was analyzed. For each of the terms identified as representatives of the various structures just described, a search was made through this corpus. The search was by word-root, so an entry could be counted regardless of whether it was adjective, noun, or verb.

We concentrated on the most objective distinctions, those that we believed could be most easily replicated by an independent investigator. The dictionaries' translations were not standardized with respect to amount of detail (with regard to how much word-roots in the original language were split into separate terms, and how many alternate translations were provided), so that a difference of one unit in occurrence-frequency in one language is not really equivalent to that in another. The most meaningful threshold, in our view, is between having at least one word (i.e., one salient enough to catch the attention of the dictionary-author and also salient in English to that author) corresponding to an English concept, versus not having one. Based on this, we judged that the best distinctions, for each term within each language, to be whether (a) a term appeared at least once, in a way clearly descriptive of a human attribute, or (b) the term appeared only in a more obliquely relevant way, or (c) the term did not appear at all. 
For each term relevant to each of our personality-relevant structures, a categorization was made as (a) or (b) or (c). ${ }^{5}$

There were a variety of ways in which a term could be obliquely relevant, fitting criterion (b). Four key ways: (i) clearly not referring to a person (e.g., 'a creative idea'), (ii) occurring as a small part of a definition that centered on another attribute-concept, (iii) representing a highly contextualized expression of the attribute (e.g., 'irritated by mosquitoes', 'sexually frustrated', 'untidy handwriting', 'walks very quietly'), (iv) invoking the concept but not involving the same sense implied by the English term (e.g., 'fearsome' instead of fearful, 'understanding' in the sense of knowing things that few know rather than having sympathy). Any of these oblique uses suggests the language contains some form of the concept even if not commonly and directly applied to humans in the way the term is in English.

For present purposes, the most important issue was whether a language had a single term, salient enough to appear in the dictionary, that could be used to translate the human-attribute term from English - satisfying criterion (a) above. Or secondarily if, failing that, there was at least evidence that the language had a more indirect use of the concept, satisfying criterion (b) above. The count (number of languages) on criterion (a), or perhaps criteria (a) and (b) combined, provides an index of the degree of the relative cross-language ubiquity of the concept represented by the term. Unlike absolute ubiquity (i.e., universality), relative ubiquity is in continuous rather than discrete variation, ranging from zero (no ubiquity at all) to true ubiquity.

A hypothesis representing claims made for the Big Five model would be that terms associated with the five personality factors will have greater relative ubiquity than those for competing models. A more realistic hypothesis, given the results of Study 1, might be that terms associated with one- and two-dimensional structural models for personality attributes will have greater relative ubiquity than those with five- or six dimensions.

\section{Results}


Table 3 presents the count for each term (i.e., for its constituent word-root), arrayed within each structural model. Provided is the number of languages meeting criterion (a); the number in brackets, if any, is the count for those terms meeting criterion (b) (but not criterion [a]).

How many terms associated with each factor typically appeared in definitions of indigenous words across these 12 mutually isolated languages? On a factor-by-factor basis, the mean counts were highest for Evaluation ( 8.0 out of 12 for the briefer and 7.1 for the longer set) and for both Big Two Social Self-Regulation (8.4 briefer, 8.1 longer) and Dynamism (8.6 briefer, 7.4 longer). Consistently, for the terms most associated with these structural models, a clear majority of the languages had a word with closely related meaning.

The mean counts were systematically lower for the Big Five and Big Six, as well as the Big Three and Big Four. On a factor-by-factor basis, these ranged from 3.1 to 5.9, with one exception: For the Big Five Intellect factor the mean count was 7.0 for the brief (5-term/wordroot) set and 2.2 for the longer (17-term/word-root) set; the briefer set benefited from emphasis on the widely distributed concepts of cleverness and intelligence, while the longer set was weighed down by the inclusion of many English terms whose distribution was not wide at all (e.g., introspective, unreflective, unsophisticated). Averaging across the two sets, the Big Five Intellect factor had overall a mean count of 4.6, well within the typical range for factors from structures of three to six factors.

The systematic difference in salience (relative ubiquity) between one/two- and three-to-sixfactor structural models becomes even clearer when one aggregates across the factors within a model, and across the two variants within each model. Figure 1 plots this overall pattern of results. It shows that within each model there was some variation in relative ubiquity of marker terms, but the central tendencies were quite different. ${ }^{6}$ Terms most associated with one- and twofactor models were markedly more ubiquitous than terms most associated with three- to sixfactor models, to a degree that exceeds the $95 \%$ confidence intervals of the mean estimates. ${ }^{7}$ The obvious inference: Terms most associated with one- and two-factor model have higher relative ubiquity (mean counts on criterion [a]) than do models for three, four, five, or six factors. 
One rival hypothesis might be that broader terms (e.g., good, happy) tend to be more ubiquitous and also characteristic of broader factors like the Big Two. However, this hypothesis can be discounted. There are 168 Table 3 terms for which Hampson, Goldberg, and John (1987) reported category-breadth values, and these values correlated close to zero (non-significantly, under .07 in magnitude) with the number of languages meeting criterion (a) or criterion (a) and (b) combined. Although breadth and ubiquity sometimes co-occur, there are many terms that are narrow but ubiquitous (e.g., jealous, envious, greedy) and many that are broad but nonubiquitous (e.g., complex, conventional, extraverted).

Going outside the relative ubiquity criterion, one might ask: Do any of these languages seem to lack any term/concept related to any one of the personality factors referenced in Table 3 ? No. All languages had at least one term related to each of the Table 3 clusters. However, in a few cases, there was only one. These content-classes seem to exist in some form in all these languages, although sometimes only in threadbare form.

\section{Discussion}

Study 2 supports a clear conclusion, based on data from 12 mutually isolated languages. Core descriptors for Big One (Evaluation) and Big Two models tend to be found in a majority of the languages, whereas those for Big Three, 'Big Four', Big Five and Big Six models tend to be found in less than half. Big One and Big Two models tend to draw on more ubiquitous descriptors than do models with more factors. In moving away from the broader factors to the three- to six-factor level, one leaves behind (to an extent, leaves out) attribute content with higher ubiquity. The more factors in one's personality model, the more one seems prone to pull in culturally specific contents. Stepping beyond two factors seems especially consequential. Once a third factor is extracted, the constraints (involving orthogonality or simple structure) in common rotation algorithms appear to locate all factors in sectors of personality-attribute space that are populated by relatively less ubiquitous concepts.

Big One and Big Two models should be easier to replicate across a wide range of cultural settings: They comprise attribute-descriptors that are somewhat easier to translate and may seem 
less 'foreign' (more emic, less etic) in non-Western settings. It may prove difficult to replicate three- to six-factor models in such settings; some factors in Western-based three- to six-factor models may not appear due to insufficient semantic representation and salience. It is not that non-Western settings or traditional societies have no more than two personality factors, but rather that after two factors indigenous models' resemblance to Western models may end.

One might wonder if, in human societies, human-attribute concepts emerge in some fixed order as one goes from smaller-scale societies to more complex ones. Study 1 indicated no clear effects of complexity on abundance of attribute terms. If factor-content emerged in some fixed order, one might expect Figure 1 to have a gradual downward slope, but it simply has a single cliff between Big Two and Big Three models. Study 2 (especially Figure 1) suggests that traditional societies, regardless of their social complexity, have the semantic emphases that would enable a Big Two model to emerge easily, but not so for something like the Big Five. This suggests the hypothesis that three- to six-factor models in the research literature in good part draw on culturally specific contents of contemporary complex societies. The data in the present studies are not adequate to test this hypothesis.

A few caveats are needed. First, these conclusions are based on studies of relatively isolated languages with limited numbers of speakers (about a million, or less). They may not apply well to languages of communities that are extensively exposed to and influenced by other languages and/or involve large populations, which might be expected (as has English) to experience a dramatic increase in potentially useable vocabulary. All of the lexical studies of personality-attributes that have been published to date have involved the latter type of languagecommunity (large populations, extensive exposure). However, one should be mindful that the vast majority of the approximately 7,000 extant human languages are based in relatively small language-communities, like the ones focused on in the present studies.

A second caveat: These limitations apply to models as measured in a highly parsimonious fashion using single-word descriptors (whether adjectives, nouns, or verbs). A way to get around this limitation is to go beyond single-word descriptors and use sentence-length descriptions of 
behavior patterns. An additional advantage: there is ambiguity in some attribute-terms - with regard to whether the reference is to an observable behavior, a subjective mental state, an effect on others, or an affordance for others -- that sentence-length descriptions can resolve. There will certainly be limits to how far this work-around can help, as illustrated by difficulties with sentence-item measures of 'openness to experience' in non-Western settings (e.g., Piedmont et al., 2002). But there should be some cross-population transferability gained by using sentences.

Finally, we cannot conclude from our studies that Big Five and Big Six (and Big Three) models are overall less useful than Big One or Big Two models. The conclusions directly reference only semantic representation across a diversity of languages, with obvious implications for cross-population replicability. We address this issue of comparative overall validity below.

Hypothetically, English terms for which it is harder to find correspondent terms in farflung languages (e.g., systematic, original, introspective) may represent more culture-specific concepts than those with easier-to-find corresponding terms. But we cannot presume that low correspondence proves culture-specificity. To establish culture-specificity, a more extensive study of the translatability and context-dependence of individual terms/concepts is needed.

\section{General Discussion}

\section{Why Would a Given Attribute-Concept Be Ubiquitous?}

Universality - a species-wide constraint on variability -- in a psychological attribute is often taken to indicate biological, especially genetic, sources of variation in the attribute. Indeed, it is well-known that differences in size and stature (included among the attributes in Table 2) are strongly affected by genes, and they also reflect regularities in visual perception that are biologically grounded. One might easily argue that there is some biological basis for any ubiquitous concept in Table 2, since a constant biological basis would tend to produce crosscultural ubiquity. For example, the general good-bad distinction that cuts across most of these terms, and shows up across all languages studied, is consistent with psychophysiological models which delineate positive and negative emotion outputs from a common evaluative space with a negativity bias (Norris, Gollan, Berntson \& Cacioppo, 2010) and basic motivational neural 
circuits tuned to appetitive and aversive stimuli (Lang \& Bradley, 2010). The greater differentiation of negative affect terms is consistent with a negativity bias in adults and infants (Ohman \& Mineka, 2001; Vaish, Grossman \& Woodward, 2008). Meta-analytic evidence of discrete neural activity related to specific emotion categories also supports a biological grounding for the ubiquitous emotion concepts in the present study (Vytal \& Hamann, 2010; but see also Lindquist, Wager, Kober, Mliss-Moreau \& Barrett, 2011)

Alternatively, universality of an attribute-concept may point to universal features of human societies not driven primarily by biology. Many of the ubiquitous concepts in Table 2 might indicate generic constraints within the social environment afforded by any human group and its cultural system, constraints to which individuals within the group may dispositionally differ in their response. The ubiquity of shame (often categorized as socially constructed and not a basic emotion) in Table 2 provides a clue in this direction. It goes against the widespread assumption that the more universal emotion labels will reflect only the most biology-driven emotions.

As Ortony and Turner (1990) suggest, apparently basic emotions may not be biologically hard-wired or necessarily salient in experience, but "simply emotions that are particularly salient in the culture" or "that represent a level in an emotion hierarchy at which the emotion terms carry the most meaning in a culture (and can thus be called basic in the sense of Rosch et al., 1976)" (p. 325). Accordingly, they might be better labelled "culturally common emotions" (Ortony \& Turner, 1990, p. 326). As these suggestions imply, basic emotion categories, like any cross-cultural universal, may reflect cultural contexts as much as biology; such emotions may have adapted into a functional role within an especially wide range of cultural contexts (cf. Mesquita \& Albert, 2007). Indeed, many of the ubiquitous concepts we found may reflect functional necessities shaped by the regularities found in human social environments.

What might be culturally common features in human social environments that contribute to use of a common set of attribute terms? Any social order includes a moral order (Goodenough, 1981): A society will have moral standards (thus good and bad/evil, and thus right and wrong) and standards for proper behavior associated with social roles (thus foolishness, and shame for 
having violated norms), sometimes involving hierarchical relations (thus being disobedient is possible). There will be communication between individuals about other individuals, often involving the aforementioned norms (thus gossip). There will be attached devotion to objects and people (thus love) that could yield an exclusivity to which threats might be perceived (thus jealousy). Individuals will differ in their access to resources (thus rich and poor), and relative social status (thus the attribute of being important), leading to potential to exaggerate or diminish one's own importance (thus humility). A society provides a context that sometimes requires choices with use of reason, prudence, and foresight (thus wise versus foolish), and various capabilities to accomplish widely valued actions and goals (the absence of which is labeled unable or stupid). We suggest that cultural systems differ in the exact form taken by components like social and moral standards, processes of attachment and communication, inequality in status and resources, and the most valued capabilities. Nonetheless, our results tend to suggest they may all have some form of each of these components. We suggest the hypothesis that many of the most ubiquitous human-attribute concepts reflect these common features in human social environments, some of which are at least partially reflected in other social species.

\section{Implications for Studies of Personality and Other Attribute Concepts}

In some languages there appear to be very few commonly used terms that function as markers for the Big Five and Big Six (as well as the Big Three, and any 'Big Four'). Therefore, an indigenous lexical study of the language would be highly unlikely to reveal the full set of factors. This should not be attributed to a language-community being unable to make a given distinction, but rather to the distinction not being particularly salient, and thus not habitually made, given the socio-cultural context. Dimensions of personality do reflect objectively existent patterns of behavior, feeling, and thinking. But they will also reflect - and sometimes be organized by - schemas for whatever are considered most important criteria on which to evaluate people. In that way, dimensions reflect the primary questions to ask about another person's worth/usefulness, and these questions cannot necessarily be assumed to be invariant across 
individuals or across populations; within differing cultural contexts (due to variant standards and values) particular questions may be variously given less importance or more importance.

But not all is subject to variation. Attribute-terms, these studies suggest, vary a good deal across languages, but are anchored by a few recurrent preoccupations. These may have their origin in common environmental as well as common genetic/biological constraints, but are expressed in a minimum, common-denominator set of criteria applied to judging people: Is the person in a highly emotional state (of some basic kind)? Is s/he moral and ethical? Is s/he competent? Is s/he debilitated in some way? How big or small? How attractive (for a potential mate, etc.)? What is his/her place in the social organization (age, gender, status, wealth)? These appear to be ubiquitous, and are good candidates to be human universals. Future studies in an even wider range of languages might better confirm their universality.

Certain aspects of the conventional approach to lexical studies impede researchers' capacity to observe these consistent preoccupations. Since Allport and Odbert (1936), terms reflecting evaluations or temporary states, even sometimes abilities, and certainly physical attributes are frequently removed from consideration before data collection and analyses. Most of the "minimum set of criteria" are then off the table, unobservable. Using wider selections of variables (e.g., studies integrated by Saucier, 2009a) tends to restore the lost capacity.

The attributes of characters in folk-tales and fairy-tales tend to be characterized less in Big Five (or Big Six) terms than by using the kind of terms found in Table 2. Such characters seem prototypically to have characteristics like good, beautiful, stupid, foolish, clumsy, disobedient, jealous, evil, happy, strong, and wise, more so than Big-Five traits (outgoing, warm, irritable, well-organized, nervous, creative). Future studies might explore whether the personality attributes most ubiquitous in popular narratives correspond to those identified here.

Results, particularly those of Study 2, underline some advantages of one- and twodimensional models of personality attributes. A caveat is that these results do not necessarily bear on the validity of recent proposals of a general factor in personality (Rushton, Bons, \& Hur, 2008); studies of such a factor have to date failed to propose a canonical set of descriptors to 
index the factor. Instead, the present results bear on the validity of Osgood's evaluation terms, which of course can be applied to objects as well as people.

However, these results should not be construed as meaning that five- or six-factor models should be abandoned. Models with more factors provide more information and more predictors, and are likely to improve on a two-factor model in predictive capacity. Moreover, models with more factors provide insights into important components or subdivisions within the broader factors. Thus, we recommend continued use of five- and six-factor models, but with a tempering of extreme claims regarding the universality of their dimensions, recognizing that they likely contain culture-specific elements. It is best to be aware of their articulation with models of fewer, broader factors that have a different set of advantages (e.g., parsimony, wider cross-cultural generality, easier integration with theory). A full understanding of personality-attribute structure needs to encompass both broader and more specific factors.

\section{Summary and Conclusions}

These studies addressed the relative universality of concepts of personality, relative to other kinds of human-attribute concepts. Consistent with some prior theories, results indicate that concepts for evaluation, size, potency, and basic emotions have substantial ubiquity across languages. But evidence here suggests that all cultural contexts stress morality and competence. A follow-up study led to a further conclusion: The concepts emphasized in prominent one- and two-dimensional models of personality attributes typically have higher cross-language ubiquity than those emphasized in models with three to six dimensions. Concepts of basic emotions, as well as personality concepts related to morality, competence, and these one- and twodimensional models may be necessary components for an adequate psychological science. These concepts may have unusually wide currency for purposes of intercultural communication. 


\section{Notes}

1. Two caveats: Bold means forward and brazen whereas brave does not. And bold is commonly used as an extraversion descriptor whereas brave is not; the opposite of bravery would be cowardice, which implies fear, more related to emotional instability.

2. One might argue that Extraversion is represented in Table 2 by Strong versus Weak, Agreeableness by Good, Humble, and Love, Conscientiousness by Clean versus Disobedient, Emotional Stability by Happy versus Afraid, Angry, Ashamed, Jealous, and Troubled, and Intellect/Openness by Wise versus Stupid and Unable. However, these are mostly atypical representatives of each of the Big Five dimensions.

3. These markers are based on lexical studies in nine languages that included Maa and Senoufo, which are also utilized here. Subsequent analyses examine whether this gives a detectable advantage to the Big Two in Study 2 results. As it turns out, it does not.

4. The third language with a highly convergent three-factor solution was Croatian, but the Croatian Big Three was the only one with narrow versions of both Extraversion and Conscientiousness (see De Raad et al., 2010, Table 5). Croatian had a relatively lowconvergence version of the four-factor solution.

5. The first (senior) author identified the correspondences, except that he identified 43 terms for which the correct categorization was not an entirely obvious and straightforward count of wordroot occurrences. For these 43, all three authors made categorizations, and the median categorization among the three was adopted (i.e., the outlier[s] eliminated). For these 43 more difficult cases, the mean correlation for the counts across three raters was .73 for (a) terms only, .59 for (b) terms, and .81 for the sum of (a) and (b) counts, yielding Coefficient Alpha reliability values of $.88, .81$, and .93 , respectively.

6. Figure 1 percentages for the Big Two descriptors (only) use only 10 languages, excluding Maa and Senoufo which were included in the Big Two study of Saucier et al. (in press) that defined these Big Two descriptors. Means and confidence intervals differed little if all 12 languages were used (as in Table 3); the same inferences follow from use of 10 or 12 languages. A table showing 
Big-Two-marker frequency counts across the 10 (rather than 12) languages is available from the first author.

7. As one would expect from the pattern in this plot, an analysis of variance -- with number of dimensions as the independent variable and mean counts on criterion (a) as the dependent variable - returned a high and significant $\mathrm{F}$ ratio, $\mathrm{F}(5,487)=9.86, \mathrm{p}<.000001$, eta $=.30$. Post hoc tests (Bonferroni, LSD, Tukey) indicated that mean counts for terms from oneand two-factor models did not differ from each other, and those from three-, four-, five-, and sixfactor models did not differ from each other. The significant differences were always, and only, between one- or two-factor models and those with more than two factors. Substituting Goldberg for 7LC terms to represent Big Three and 'Big Four' factors led to very similar results, with F(5, $532)=9.72, p<.000001$, eta $=.29$. Repeating either version of this analysis using the sum of counts for criteria (a) and (b) together, instead of criterion (a) alone, as the dependent variable, led to similar results, with F values over 8.11, $\mathrm{p}<.000001$, and eta values over .26. 


\section{References}

Allik, J., Realo, A., \& McCrae, R. R. (2013). Universality of the five-factor model of personality. In T. A. Widiger \& P. T. Costa (Eds.), Personality disorders and the five-factor model of personality ( $3^{\text {rd }}$ ed.). Washington, DC: American Psychological Association.

Allport, G. W., \& Odbert, H. S. (1936). Trait names: A psycho-lexical study. Psychological Monographs, 47, (1, Whole No. 211).

Brown, D. E. (1991). Human universals. Philadelphia: Temple University Press.

Buss, D. M. (2008). Human nature and individual differences: Evolution of human personality. In O. P. John, R. W. Robins, \& L. A. Pervin (Eds.), Handbook of personality: Theory and research ( $3^{\text {rd }}$ ed., pp. 29-60). New York: Guilford.

Calabrese, W. R., Rudick, M. M., Simms, L. J., \& Clark, L. A. (2012). Development and validation of Big Four personality scales for the Schedule for Nonadaptive and Adaptive Personality - Second Edition (SNAP-2). Psychological Assessment, 24, 751-763.

Capell, A. (1968). A new Fijian dictionary ( $3^{\text {rd }}$ ed.). Suva, Fiji: Obtainable at the government printer.

Carlson, R. (undated). Supyire dictionary. Draft version.

Cheung, F. M., van de Vijver, F.J. R, \& Leong, F.T.L. (2011). Toward a new approach to the study of personality in culture. American Psychologist.

Church, A. T. (2008). Current controversies in the study of personality across cultures. Social and Personality Psychology Compass , 2, I930-I951.

Cuddy, A.J.C., Fiske, S.T., Kwan, V.S.Y., Glick, P., Demoulin, S., Leyens, J. Ph. , \& Bond, M.H. (2009). Stereotype content model across cultures: Universal similarities and some differences. British Journal of Social Psychology 48, 1-33. 
DeYoung, C. G. (2006). Higher-order factors of the Big Five in a multi-informant sample. Journal of Personality and Social Psychology, 91, 1138-1151.

DeYoung, C. G. (2010). Toward a theory of the Big Five. Psychological Inquiry, 21, 26-33.

De Raad, B., Barelds, D. P. H., Levert, E., Ostendorf, F., Mlacic, B., Di Blas, L., Hrebickova, M., Szirmak, Z., Szarota, P., Perugini, M., Church, A.T., \& Katigbak, M. S. (2010). Only three factors of personality description are fully replicable across languages: A comparison of 14 trait taxonomies. Journal of Personality and Social Psychology, 98, 160-173.

Di Blas, L., \& Forzi, M. (1998). An alternative taxonomic study of personality descriptors in the Italian language. European Journal of Personality, 12, 75-101.

Dixon, R. M. W. (1982). Where have all the adjectives gone? and other essays in semantics and syntax. New York: Mouton.

Ekman, P., Sorenson, E. R., \& Friesen, W. V. (1969, April 4). Pan-cultural elements in facial displays of emotion. Science, 164, 86-88.

Fish, S. E. (1999). The trouble with principle. Cambridge, MA: Harvard University Press.

Fiske, S. T., Cuddy, A. J. C., \& Glick, P. (2006). Universal dimensions of social cognition: Warmth and competence. Trends in Cognitive Sciences, 11, 77-83.

Francis, W. N., \& Kucera, H. (1982). Frequency analysis of English usage. Boston: Houghton Mifflin.

Georgas, J. \& Mylonas, K. (2006). Cultures are like all other cultures, like some other cultures, like no other culture. In U. Kim, K.-S. Yang, \& K.-K. Hwang (Eds.), Indigenous and cultural psychology: Understanding people in context (pp. 197-221). New York: Springer. 
Goldberg, L. R. (1981). Language and individual differences: The search for universals in personality lexicons. In L. Wheeler (Ed.), Review of personality and social psychology, vol. 2 (pp. 141-165). Beverly Hills, CA: Sage.

Goldberg, L. R. (1992). The development of markers for the Big Five factor structure. Psychological Assessment, 4, 26-42.

Goldberg, L. R. (1993). The structure of phenotypic personality traits. American Psychologist, $48,26-34$.

Goldberg, L. R., Johnson, J. A., Eber, H. W., Hogan, R., Ashton, M. C., Cloninger, C. R., \& Gough, H. C. (2006). The International Personality Item Pool and the future of publicdomain personality measures. Journal of Research in Personality, 40, 84-96.

Goodenough, W. H. (1981). Culture, language, and society (2 ${ }^{\text {nd }}$ ed.). Menlo Park, CA: Benjamin/Cummings.

Greenberg, J. H. (1966). Language universals. The Hague, the Netherlands: Mouton. Greenberg, J. H. (1975). Research on language universals. Annual Review of Anthropology, 4, $75-94$.

Haacke, W. H. G., \& Eiseb, E. (2002). A Khoekhoegowab dictionary with an EnglishKhoekhoegowab index. Windhoek, Namibia: Gamsberg Macmillan.

Haidt, J. (2007), The new synthesis in moral psychology. Science, 316 (5827), 998-1002.

Hamlin, J.K., Wynn, K. \& Bloom, P. (2010). Three-month-old infants show a negativity bias in social evaluation. Developmental Science, 13, 923-929.

Harter, S. (2001). The cognitive and social construction of the developing self. New York: Guilford Press.

Heimbach, E. E. (1979). White Hmong - English dictionary. Linguistic series IV, data paper number 75. Ithaca, NY: Southeast Asia Program Publications. 
Heine, S. J., \& Buchtel, E. E. (2009). Personality: The universal and culturally specific. Annual Review of Psychology, 60, 369-394.

Holmer, N. M. (1952). Ethno-linguistic Cuna dictionary with indices and references to a critical and comparative Cuna grammar (Etnologiska studier 14). Göteborg: Etnografiska Museet.

The Hopi Dictionary Project (1998). Hopi dictionary / Hopìikwa lavàytutuveni: A Hopi-English dictionary of the Third Mesa Dialect. Tucson, AZ: University of Arizona Press.

Hupka, R. B., Lenton, A. P., \& Hutchison, K. A. (1999). Universal development of emotion categories in natural language. Journal of Personality and Social Psychology, 77, 247278.

Izard, C. E. (1992). Basic emotions, relations among the emotions, and emotion-cognition relations. Psychological Review, 99, 562-565.

Judd, C. M., James-Hawkins, L., Yzerbyt, V., \& Kashima, Y. (2005). Fundamental dimensions of social judgement: Understanding the relations between judgments of competence and warmth. Journal of Personality and Social Psychology, 89, 899-913.

Kelly, G. A. (1955). The psychology of personal constructs. New York: Norton.

Kilham, C. A., et al. (1976). Dictionary and source-book of the Wik-Mungkan language. Darwin, Australia: Summer Institute of Linguistics.

Lang, A. (1973). Enga dictionary with English index (Pacific Linguistic, Series C, No. 20). Canberra: Department of Linguistics, Research School of Pacific Studies, the Australian National University. 
Leach, C. W., Ellemers, N., \& Barreto, M. (2007). Group virtue: The importance of morality (versus competence and sociality) in the positive evaluations of ingroups. Journal of Personality and Social Psychology, 93, 234-249.

Lee, K., \& Ashton, M. C. (2008). The HEXACO personality factors in the indigenous lexicons of English and 11 other languages. Journal of Personality, 76, 1001-1053

Lindquist, K. A., Wager, T. D., Kober, H., Bliss-Moreau, E., Barrett, L. F. (in press). The brain basis of emotion: A meta-analytic review. Behavioral and Brain Sciences.

Lorrain, R. A. (1951). Grammar and dictionary of the Lakher or Mara language. Gauhati, India: Government of Assam in the Department of Historical and Antiquarian Studies.

McCrae, R. R., \& Costa, P. T. (1997). Personality trait structure as a human universal. American Psychologist, 52, 509-516.

Mesquita, B., \& Albert, D. (2007). The cultural regulation of emotions. In J. J. Gross (Ed.), Handbook of emotion regulation (pp. 486-503). New York: Guilford.

Murdock, G. P. (1945). The common denominator of cultures. In R. Linton (Ed.), The science of man in the world crisis (pp. 123-142). New York: Columbia University Press.

Murdock, G. P. (1967). Ethnographic atlas. Pittsburgh: University of Pittsburgh Press.

Norenzayan, A., \& Heine, S. J. (2005). Psychological universals: What are they and how can we know? Psychological Bulletin, 131, 763-784.

Norris, C. J., Golan, J., Berntson, G. G., \& Cacioppo, J. T. (2010). The current status of research on the structure of evaluative space. Biological Psychology, 84, 422-436.

Ortony, A., \& Turner, T. J. (1990). What's basic about basic emotions? Psychological Review, 97, 315-331.

Osgood, C. E. (1962). Studies on the generality of affective meaning systems. American Psychologist, 17, 10-28. 
Osgood, C. E., May, W., \& Miron, M. (1975). Cross-cultural universals of affective meaning. Urbana: University of Illinois Press.

Parker, E. M., \& Hayward, R. J. (1985). An Afar-English-French dictionary (with grammatical notes in English). London: School of Oriental and African Studies, University of London.

Payne, D. L., \& Ole-Kotikash, L. (2003). Maa lexicography and text project. Draft version.

Piedmont, R. L., Bain, McCrae, R. R. \& Costa, P. T. (2002). The applicability of the Five-Factor Model in a sub-Saharan culture. In R. R. McCrae \& J. Allik (Eds.), The five-factor model of personality across cultures (pp. 155-173). New York: Kluwer Academic/Plenum.

Plutchik, R. (1980). Emotion: A psychoevolutionary analysis. New York: Harper \& Row.

Raybeck, D., \& Herrmann, D. (1996). Antonymy and semantic relations: The case for a linguistic universal. Cross-Cultural Research, 30, 154-183.

Rosch, E. H., Mervis, C. B., Gray, W. D., Johnson, D. M., \& Boyes-Braem, P. (1976). Basic objects in natural categories. Cognitive Psychology, 8, 382-439.

Rosenberg, S., Nelson, C., \& Vivekananthan, P. S. (1968). A multidimensional approach to the structure of personality impressions. Journal of Personality and Social Psychology, 9, 283-294.

Rozin, P., Berman, L., \& Royzman, E. (2010). Biases in use of positive and negative words across twenty natural languages. Cognition, 24, 536-548.

Saucier, G. (2008). Measures of the personality factors found recurrently in human lexicons. In G. J. Boyle, G. Matthews, \& D. Saklofske (Eds.), Handbook of personality theory and testing: Vol. 2, Personality measurement and assessment (pp. 29-54) London: Sage.

Saucier, G. (2009a). Recurrent personality dimensions in inclusive lexical studies: Indications for a Big Six structure. Journal of Personality, 77, 1577-1614. 
Saucier, G., \& Goldberg, L. R. (2001). Lexical studies of indigenous personality factors:

Premises, products, and prospects. Journal of Personality, 69, 847-879.

Saucier, G. (2009b). What are the most important dimensions of personality? Evidence from studies of descriptors in diverse languages. Personality and Social Psychology Compass, $3,620-637$.

Schmitt, D., Allik, J., McCrae, R.R., Benet-Martinez, Alcalay, L, Ault, L., et al. (2007). The geographic distribution of Big Five personality traits: Patterns and profiles of human selfdescription across 56 nations. Journal of Cross-Cultural Psychology, 38: 173-212.

Schneider, L. (1985). Ulírnaísígutiit: An Inuktitut-English dictionary of northern Quebec, Labrador, and Eastern Arctic dialects (with an English-Inuktitut index) (D. R. F. Collis, trans.). Québec: Les Presses de l’Université Laval.

Schonell, F. J., Meddleton, I. G., \& Shaw, B. A. (1956). A study of the oral vocabulary of adults: An investigation into the spoken vocabulary of the Australian worker. Brisbane, Australia: University of Queensland Press.

Service, E. R. (1971). Primitive social organization: An evolutionary perspective ( $2^{\text {nd }}$ ed.). New York: Random House.

Solomon, R. C. (2002). Back to basics: On the very idea of 'basic emotions.' Journal for the Theory of Social Behavior, 32, 115-144.

Tomkins, S. S. (1970). Affect as the primary motivational system. In M. B. Arnold (Ed.), Feelings and emotions (pp. 101-110). New York: Academic Press.

Tooby, J., \& Cosmides, L. (2000). Toward mapping the evolved functional organization of the mind and brain. In M. S. Gazzaniga (Ed.), The new cognitive neurosciences (2nd ed., pp. 1167-1178). Cambridge, MA: MIT Press. 
Vaish, A., Grossmann, T., \& Woodward, A. (2008). Not all emotions are created equal: The negativity bias in early social-emotional development. Psychological Bulletin, 134, 383403.

Vytal, K., \& Hamann, S. (2010). Neuroimaging support for discrete neural correlates of basic emotions: A voxel-based meta-analysis. Journal of Cognitive Neuroscience, 12, 28642885.

Wierzbicka, A. (1996). Semantics: Primes and universals. Oxford, UK: Oxford University Press.

Wojciszke, B. (2005a). Affective concomitants of information on morality and competence. European Psychologist, 10, 60-70.

Wojciszke, B. (2005b). Morality and competence in person- and self-perception. European Review of Social Psychology, 16, 155-188. 
Table 1

The Twelve Languages Studied

\begin{tabular}{|c|c|c|c|c|c|c|}
\hline \multirow[b]{2}{*}{ Language } & \multirow[b]{2}{*}{ Language Family } & \multirow[b]{2}{*}{ Dictionary } & \multirow[b]{2}{*}{ Region } & \multicolumn{3}{|c|}{ Number of Dictionary Entries } \\
\hline & & & & Total & Human-Attribute & $\%$ \\
\hline Wik-Mungkan & Australian & Kilham et al. (1976) & Australia & 4,200 & 382 & 9 \\
\hline Inuktitut & Eskimo-Aleut & Schneider (1985) & Arctic America & 21,300 & 1816 & 9 \\
\hline Khoekhoe & Khoisan & Haacke \& Eiseb (2002) & Southern Africa & 24,500 & 2592 & 11 \\
\hline Maa & Nilotic & Payne \& Ole-Kotikash (2003) & East Africa & 5,600 & 779 & 14 \\
\hline Enga & Trans-New-Guinea & Lang (1973) & New Guinea & 5,200 & 507 & 10 \\
\hline Kuna & Chibchan & Holmer (1992) & Central/South America & a 6,100 & 306 & 5 \\
\hline Hmong (White) & Hmong-Mien & Heimbach (1979) & Southeast Asia & 5,100 & 946 & 19 \\
\hline Hopi & Uto-Aztecan & Hopi Dictionary Project (1998) & North America & 30,000 & 2725 & 9 \\
\hline Afar & Afro-Asiatic & Parker \& Hayward (1985) & North Africa & 9,300 & 3455 & 37 \\
\hline Fijian & Austronesian & Capell (1991) & Pacific Islands & 5,800 & 1320 & 23 \\
\hline Mara Chin (Lakher) & Sino-Tibetan & Lorrain (1951) & South Asia & 7,000 & 1351 & 19 \\
\hline Supyire (Senoufo) & Niger-Congo & Carlson (undated) & West Africa & 5,500 & 678 & 12 \\
\hline
\end{tabular}

Note. Languages are ordered based on relatively degree to which egalitarian band (vs. hierarchical chiefdom) organization is present (based on Service [1971], see Appendix) 
Table 2

$\underline{\text { The Most Ubiquitous Concepts Referring to Human Attributes }}$

Found in All 12 Languages

Found in 11 of 12 Languages

Concept Concept Language in which missing

Evaluative concepts referring primarily to moral character

Bad Evila Kuna

Good

Evaluative concepts referring primarily to competence

Useless $s_{b}$

Evaluative concepts referring primarily to physical appearance

Beautiful $_{\mathrm{c}}$ Handsome Kuna

Behavioral propensities relevant to moral character

Disobedient $_{\mathrm{d}}$

$\begin{array}{ll}\text { Gossip }_{\mathrm{e}} & \text { Kuna } \\ \text { Humble }_{\mathrm{f}} & \text { Kuna } \\ \text { Love }_{\mathrm{g}} & \text { Enga }\end{array}$

Behavioral propensities relevant to competence

Stupid

Clumsy

Mara Chin

Drunk

Wik-Mungkan

Foolish Kuna

Quick Enga

Slow Enga

Unable $_{\mathrm{h}} \quad$ Wik-Mungkan

Wise Wik-Mungkan

Potency/debility concepts (indirectly relevant to competence; includes health status)

Alive $_{\mathrm{i}}$

Blind

Dead

Kuna

Sick

Sleepy

Enga

Strong $_{j}$

Tired

Weak $_{\mathrm{k}}$

Well $_{1}$

Motivational state concepts

$\begin{array}{ll}\text { Hungry } & \text { Mara Chin } \\ \text { Pain }_{\mathrm{m}} & \text { Kuna } \\ \text { Pleasure }_{\mathrm{n}} & \text { Wik-Mungkan } \\ \text { Thirsty } & \text { Kuna }\end{array}$


Table 2 (continued)

The Most Ubiquitous Concepts Referring to Human Attributes

Found in All 12 Languages

Found in 11 of 12 Languages

Concept

Concept

Language in which missing

\begin{tabular}{|c|c|c|}
\hline \multicolumn{3}{|c|}{ Emotion concepts } \\
\hline Afraid & Happy & Enga \\
\hline Angry & Satisfied $_{o}$ & Wik-Mungkan \\
\hline Ashamed $_{\mathrm{p}}$ & Troubled & Kuna \\
\hline \multicolumn{3}{|l|}{ Jealous $_{\mathrm{q}}$} \\
\hline \multicolumn{3}{|l|}{ Surprised } \\
\hline \multicolumn{3}{|c|}{ Size (physical appearance) concepts } \\
\hline Big & $\mathrm{Fat}_{\mathrm{r}}$ & Kuna \\
\hline Large & Little & Wik-Mungkan \\
\hline Small & Short & Kuna \\
\hline Heavy $_{\mathrm{s}}$ & Tall & Kuna \\
\hline \multicolumn{3}{|c|}{ Age and social-position (social status or role) concepts } \\
\hline Old & Married $_{t}$ & Kuna \\
\hline \multirow[t]{3}{*}{ Young } & Poor & Wik-Mungkan \\
\hline & Rich & Kuna \\
\hline & Stranger $_{\mathrm{u}}$ & Mara Chin \\
\hline \multicolumn{3}{|c|}{ Potentially relevant, but not always clear whether the term can refer to humans } \\
\hline $\operatorname{Cold}_{\mathrm{v}}$ & Clean & Enga \\
\hline Dirty $_{w}$ & Crooked & Inuktitut \\
\hline Hard & Different & Hmong \\
\hline $\operatorname{Hot}_{\mathrm{x}}$ & Important & Kuna \\
\hline & Lost & Kuna \\
\hline & Peace(ful) y & Kuna \\
\hline & Right & Inuktitut \\
\hline & Rotten & Supyire \\
\hline & Smell(y) & Hmong \\
\hline & Soft & Kuna \\
\hline & Straight & Kuna \\
\hline & Wrong & Kuna \\
\hline
\end{tabular}

Notes.

a. Synonym "Wicked" found in Kuna and 9 other languages

b. In Supyire not "useless" but rather a term meaning "utility/usefulness."

c. Clearly refers to men as well as women in many of the languages; antonym "ugly" not found in Hmong, Kuna, Mara Chin.

d. Disobedient more common than obedient, but Hopi had only obedient.

e. Appears in various forms, but adjective form ("Gossipy") rare 
Table 2 (continued)

The Most Ubiquitous Concepts Referring to Human Attributes

f. Antonym "Proud" not found in Kuna, Supyire, Wikmungkan.

g. Antonym "Hate" not found in Enga or 3 other languages

h. Antonym "Able" also found in all but Wik-Mungkan.

i. "Alive" not found in Kuna or Khoekhoe, but concept of "life" is.

j. Often synonymous with powerful or brave or clever/capable or healthy.

k. Often synonymous with soft, tired, or sick; sometimes with helpless or unable to walk.

1. In Khoekhoe, senses are "well-being" and "in good condition."

m. Usually in noun form ("pain" or "in pain").

n. Usually "pleased"; in Supyire only "pleasing" is evident

o. In Fijian only "dissatisfied" appeared

p. Includes any term from the root "shame"

q. In most languages at least one term means both jealous, envious.

r. Antonym "Thin" found in all but Kuna and Wik-Mungkan.

s. Sometimes synonymous with difficult, pregnant, or slow; antonym "light (in weight)" only appeared in seven of 12 languages.

t. Often "unmarried"; Kuna does have term for "marriageable."

u. In Mara Chin one term means "barbarian/foreigner/alien."

v. Physical meaning ubiquitous, characterological meaning occasional.

w. Often synonymous with disgusting, sometimes with impure, untidy, slovenly, or obscene.

$\mathrm{x}$. In most languages at least one of the terms for hot is synonymous with "angry, irritable";

sometimes synonymous with zest/zeal.

y. h. In Enga and Fijian, unclear if term refers only to collectives. 
Table 3

$\underline{\text { Relative Ubiquity of Terms Representing Various Lexically-based Structural Models }}$

\section{Evaluation}

Short set (mean 8.0 [0.6]): Good (12), -Bad (12), Beautiful (12), -Ugly (9), Nice (5 [2]), Pleasant (5 [1]), -Awful (1 [1])

Longer set (mean 7.1 [1.1]) adds: Happy (10 [1]), Kind (10), Friendly (9), -Worthless (9), Useful (8 [3]), -Dangerous (7 [3]), Comfortable (6 [3]), -Cruel (6 [1]), -Despicable (6 [1]), Superior (4 [3]), -Inferior (3 [1]), Lovely (1 [1])

\section{Big Two Social Self-Regulation}

Short set (mean 8.4 [0.5]): Good (12), Obedient (12), Kind (10), Generous (9), Respectful (9), Honest (8), -Selfish (8), Diligent (6), Gentle (5 [3]), Responsible (5 [2])

Longer set (mean 8.1 [0.5]) includes the above as well as: Careful (10 [1]), Calm (9), Patient (9), Polite (9), Disciplined (0 [1])

\section{Big Two Dynamism}

Short set (mean 8.6 [0.0]): -Weak (12), Bold (10), Brave (10), -Shy (8), -Timid (8), Active (6), Lively (6)

Longer set (mean 7.4 [0.1]) includes the above as well as: Strong (12), -Fearful (11), -Sad (10), Silent (9), Energetic (8 [1]), -Cowardly (8), Daring (5), Sociable (5), Cheerful (4 [1]), Pessimistic (2), Dynamic (0)

\section{Big Three Conscientiousness}

For narrow-interpretation set, see 7LC Big Five Conscientiousness below (mean 5.5 [0.3]). Broad-interpretation set (mean 4.25 [0.6]): -Careless (10 [1]), Industrious (6 [1]), Diligent (6), Untidy (6), Responsible (5 [2]), -Absent-minded (5), -Unstable (5), Rational (3), -Reckless (3), Organized (2), Methodical (0 [2]), Disciplined* (0 [1])

\section{Big Three Agreeableness}

For narrow-interpretation set, see 7LC Big Five Agreeableness below (mean 4.0 [0.4].

Broad-interpretation set (mean 5.5 [1.7]): Kind (10), Loving (9 [3]), Friendly (9), Generous (9), -Selfish (8), -Arrogant (6), -Rude (4 [1]), Sympathetic (4), -Vindictive (3), Good-natured (3), Cold (1 [11]), -Warm (0 [5]) 


\section{Big Three Extraversion}

For narrow-interpretation set, see 7LC Big Five Extraversion below (mean 3.9 [0.1])

Broad-interpretation set (mean 3.5 [0.2]): -Fearful (11), -Shy (8), -Anxious (6 [1]), Cheerful (4

[1]), -Bashful (4), -Reserved (3), Optimistic (2), -Pessimistic (2), -Introverted (2), Extraverted

(1), -Insecure (1), -Passive (1), Dynamic (0)

\section{'Big Four' Conscientiousness}

For narrow-interpretation set, see 7LC Big Five Conscientiousness below (mean 5.5 [0.3]).

Broad-interpretation set (mean 4.1 [0.8]): -Careless (10 [1]), Hard-working (8 [2]), Serious (6

[2]), -Untidy (6), Responsible (5 [2]), -Absent-minded (5), Stable (5), Persistent (4), Strict (3

[1]), Rational (3), -Reckless (3), Organized (2), Conscientious (2), Methodical (0 [2]),

Disciplined* (0 [1])

\section{'Big Four' Agreeableness}

For narrow-interpretation set, see 7LC Big Five Agreeableness below (mean 4.0 [0.4].

Broad-interpretation set (mean 5.7 [0.5]): Peaceful (10 [1]), Kind (10), Generous (9), -Selfish (8), -Arrogant (6), Gentle (5 [3]), -Aggressive (4 [1]), Sympathetic (4), -Vindictive (3), Goodnatured (3), Accommodating (1)

\section{'Big Four' Extraversion}

For narrow-interpretation set, see 7LC Big Five Extraversion below (mean 3.9 [0.1].

Broad-interpretation set (mean 3.2 [0.3]): -Shy (8), Chatty (6 [1]), Talkative (6), Sociable (5), Cheerful (4 [1]), -Bashful (4), -Reserved (3), -Antisocial (3), -Introverted (2), Expressive (1 [2]), Extraverted (1), Spontaneous (1), -Passive (1), Dynamic (0)

\section{'Big Four' Emotional Stability}

For narrow-interpretation set, see 7LC Big Five Emotional Stability below (mean 3.1 [1.6]. Broad-interpretation set (mean 5.5 [0.7]): -Fearful (11), Brave (10), Courageous (9 [1]), Clever (9 [1]), -Anxious (6 [1]), -Complaining (6 [1]), Independent (5 [1]), (Self-)Assured (4 [2]), Whiny (4), Ingenious (3), -Emotional (2 [2]), Optimistic (2), -Insecure (1),

\section{Big Five Conscientiousness}

7LC set (mean 5.5 [0.3]): Careful (10 [1]), -Lazy (10), -Negligent/Neglectful (8 [1]), Thoughtless (8), Diligent (6), -Unstable (5), Precise (4 [1]), -Inconsistent (4), Thorough (3), Conscientious (2), Systematic (0) 
Goldberg set (mean 3.7 [0.5]): Careful* (10 [1]), -Negligent* (8 [1]), Efficient (7), Neat (5), Steady (4 [3]), -Inconsistent* (4), -Sloppy (3 [1]), -Haphazard (3), Thorough* (3), -Undependable (3), Conscientious* (2), Organized (2), Practical (1 [2]), Prompt (1), Systematic* (0)

\section{Big Five Agreeableness}

7LC set (mean 4.0 [0.4]): Peaceful (10 [1]), -Aggressive (4 [1]), -Egotistical/Egocentric (4), Helpful (3 [1]), -Domineering (3), -Bossy (2), Tolerant (2)

Goldberg set (mean 4.6 [1.5]): Kind (10), Generous (9), -Selfish (8), Agreeable (7 [1]), Pleasant (5 [1]), -Uncharitable (5), Trustful (4 [2]), Cooperative (4 [1]), -Demanding** (4 [1]), Rude (4 [1]), Sympathetic (4), Helpful* (3 [1]), Considerate (3), -Harsh (3), -Cold (1 [11]), Warm (0 [5])

\section{Big Five Emotional Stability}

7LC set (mean 3.1 [1.6]): -Anxious (6 [1]), -Hyper[or Over]sensitive (4 [3]), -Nervous (4 [2]), (Self-)Assured (4 [2]), -Emotional (2 [2]), Imperturbable (2 [1]), -Vulnerable (0)

Goldberg set (mean 4.6 [0.9]): -Jealous (12), -Fearful (11), -Envious (9), -Irritable (8), Anxious* (6 [1]), Relaxed (5 [1]), -Nervous* (4 [2]), Undemanding** (4 [1]), -Touchy (4 [1]), Fretful (3), -Insecure (1), -Moody (5), Unexcitable (3), -Emotional* (2 [2]), Imperturbable* (2 [1]), -Self-Pitying (2 [7]), -High-strung (0), -Temperamental (1 [1])

\section{Big Five Extraversion}

7LC set (mean 3.9 [0.1]): -Silent (9), Energetic (8 [1]), -Shy (8), -Timid (8), Talkative (6), Sociable (5), -Bashful (4), -Reserved (3), -Introverted (2), -Withdrawn (2), Extraverted (1), Passive (1), Vivacious (1), Dynamic (0), Temperamental** (1 [1])

Goldberg set (mean 4.4 [0.3]): Bold (10), Energetic* (8 [1]), -Quiet (8 [1]), -Shy* (8), Timid* (8), Active (6), Talkative* (6), Unrestrained (5 [2]), Daring (5), -Bashful (4), Vigorous (4), -Reserved* (3), -Inhibited (2), -Introverted* (2), -Withdrawn* (2), Extraverted* (1), Verbal, Assertive (0 [2]), Unadventurous (0)

\section{Big Five Intellect}

7LC set (mean 7.0 [0.8]): Intelligent (10), Clever (9 [1]), -Un[der]educated (7 [1]), Knowledgeable (6 [1]), Imaginative (3 [1])

Goldberg set (mean 2.2 [1.5]): -Unintelligent* (10), Bright (5 [2]), -Uninquisitive (4),

Simple (3 [3]), Imaginative* (3 [1]), Creative (3), -Imperceptive (3), Intellectual (2 [4]), Deep (2 [9]), Artistic (1 [1]), Philosophical (1 [1]), -Unsophisticated (1), -Shallow (0 [3]), Complex (0, 2), Innovative (0), Introspective (0), -Unreflective (0) 


\section{Big Six Conscientiousness}

CL6 set (mean 4.4 [0.6]): -Careless (10 [1]), -Lazy (10), Orderly (8), Industrious (6 [1]), Diligent (6), -Irresponsible (5 [2]), -Rash (5 [1]), -Absent-minded (5), Precise (4 [1]), $\quad$-Frivolous (4), -Reckless (3), Thorough (3), Conscientious (2), Organized (2), Meticulous (1), Dutiful (1 [4]), Disciplined (0 [1])

WCL6 set (mean 3.5 [0.5]): Orderly (8), Tidy (6), Responsible* (5 [2]), Neat (5), Consistent (4), Hard-working (8 [2]), Organized* (2), Meticulous (1), Disciplined* (0 [1]), Moderate (0 [1]), Systematic (0)

\section{Big Six Honesty (and Humility or Propriety)}

CL6 set (mean 5.4 [0.3]): -Greedy (9), Just (9), Honest (8), -Boastful (7 [2]), -Hypo-critical (7), Sincere (5 [1]), -Sly (4), -Conceited (3), Loyal (2), -Calculating (0)

WCL6 set (mean 5.9 [0.8]): -Bad (12), -Evil (11), -Wicked (9 [1]), -Dangerous (7 [3]), Insane (7), -Cruel (6 [1]), -Disgusting (6 [3]), -Corrupt (5 [1]), -Vicious (4), -Inhuman (2), Awful (1 [1]), -Beastly (1)

\section{Big Six Agreeableness}

CL6 set (mean 5.0 [0.8]): Peaceful (10 [1]), -Stubborn (9 [1]), Patient (9), -Irritable (8), Agreeable (7 [1]), -Aggressive (4 [1]), Good-natured (3), -Choleric (2), Tolerant (2), -Hotheaded (5 [6]), Mild (1), -Authoritarian (0)

WCL6 set (mean 5.3 [0.9]): Peaceful* (10 [1]), Kind (10), -Stubborn* (9 [1]), Calm (9), Generous (9), -Impatient* (9), -Irritable* (8), -Anxious** (6 [1]), -Quick-tempered (6 [1]), Gentle (5 [3]), -Quarrelsome (4 [1]), Easygoing (3), -Irascible (2), Tolerant* (2), -Brawling (1 [1]), -Fiery (1), -Impulsive (1), Understanding (0 [8])

\section{Big Six Emotionality vs. Resiliency}

CL6 set (mean 4.6 [0.9]): -Strong (12), -Brave (10), -Courageous (9 [1]), Anxious** (6 [1]), Independent (5 [1]), -(Self-)Assured (4 [2]), -Resolute (4), Emotional (2 [2]), -Imperturbable (2 [1]), Fragile (1 [3]), Sentimental (0), Vulnerable (0)

WCL6 set (mean 5.7 [0.8]): Fearful (11), Sad (10), Cowardly (8), Depressed (4 [1]), Frustrated (1 [2]), Gloomy (0 [2])

\section{Big Six Extraversion}


CL6 set (mean 4.1 [0.3]): -Silent (9), -Quiet (8 [1]), -Shy (8), Lively (6), Talkative (6), Sociable (5), Cheerful (4 [1]), -Solitary (3 [3]), -Reserved (3), -Introverted (2), -Taciturn (2), -Withdrawn (2), Extraverted (1), -Passive (1), Vivacious (1) WCL6 set (mean 4.1 [0.1]): Friendly (9), -Silent* (9), Talkative* (6), Sociable* (5), Cheer-ful* (4 [1]), Smiling (3), Outgoing (2), -Withdrawn* (2), Vivacious* (1), Gregarious (0)

\section{Big Six Openness or Originality}

CL6 set (mean 3.4 [1.2]): Intelligent (10), Clever (9 [1]), Sharp (5 [4]), Creative (3), Intel-lectual (2 [4]), Gifted (2 [2]), Ironic (2 [1]), -Conservative (1), -Conventional (0), Original (0) WCL6 set (mean 3.5 [1.7]): Wise (11), Intelligent* (10), Important (7 [4]), Knowledgeable (6 [1]), Outstanding (4), Admirable (3 [3]), Intellectual* (2 [4]), -Traditional (2 [3]), Talented (3 [1]), Impressive (2), -Ordinary (1 [4]), Unusual (1 [3]), Brilliant (1 [1]), -Average (0 [2]), Original $(0)$

Note. Number in parentheses gives the number of languages (out of 12) in which at least one indigenous term met criterion (a). Number in brackets gives the number, if any, of languages in which at least one indigenous term met criterion (b) although none met criterion (a) in that language. 7LC - seven-language core terms for Big Five derived from De Raad et al. (1998). CL6 - Cross-Language Six, terms derived from Ashton et al. (2004). WCL6 - Wide-variable-selection Cross-Language Six, terms from Saucier (2009a). * Term also appearing on other model with same number of factors, on corresponding factor. ${ }^{* *}$ Term also appearing on other model with same number of factors, but on a different factor. Terms are given in adjective form, but expression in any word-form (including nouns and verbs) was counted. The count for each term refers to its word-root across all word forms, and not only to its appearance in adjective form. 
Figure 1. Error bars (showing 95\% confidence intervals around each mean) for means on criterion (a), categorized by number of dimensions in the structural model.

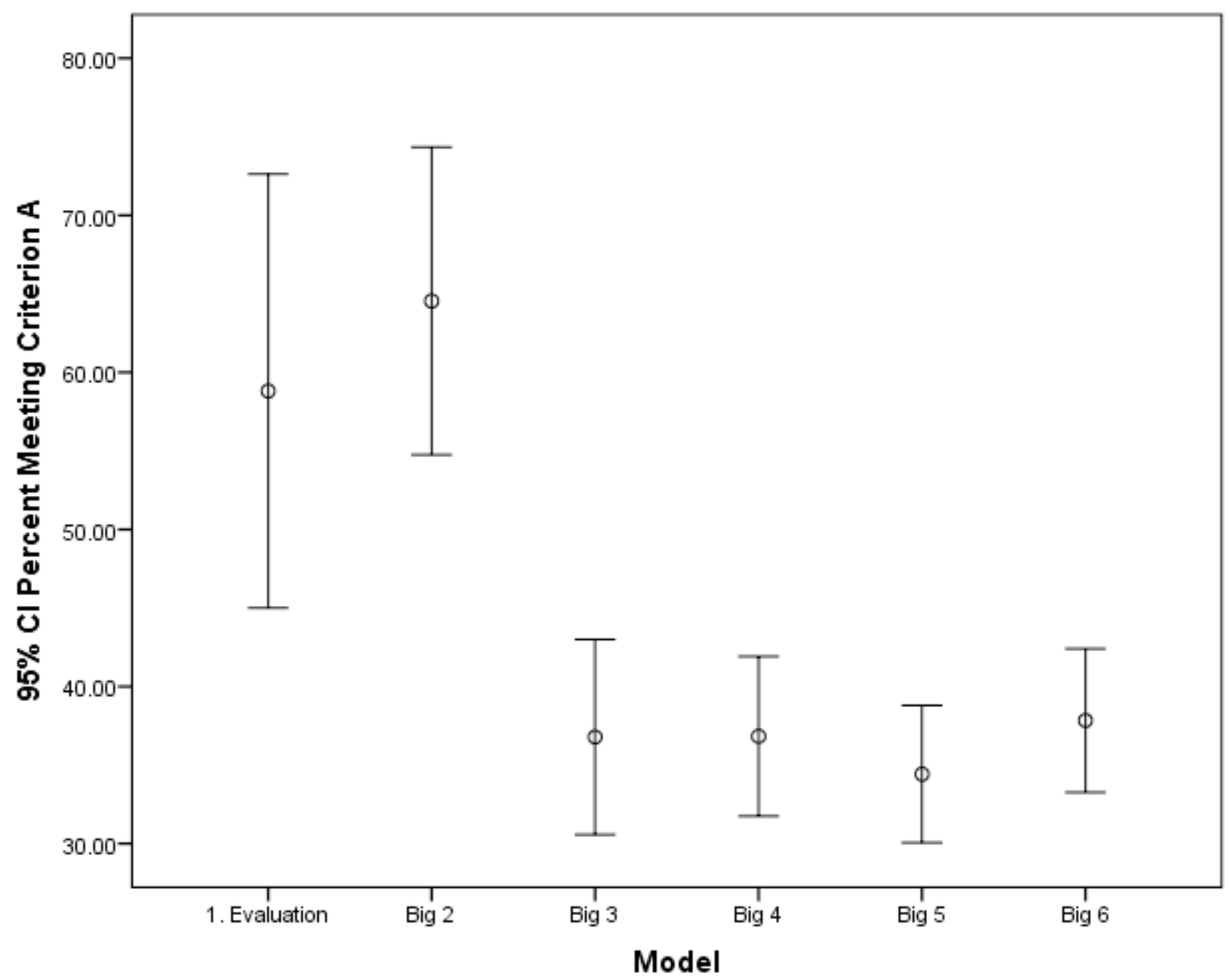


Appendix

Most Distinctive Ethnographic Features of the Traditional Culture Associated with Each Language (and Indications of Position on Service's Band-Tribe-Chiefdom Continuum)

Khoekhoe. Hunting/husbandry, migratory, occasional polygyny, hereditary headman succession, wealth distinctions, no individual land rights. (A, B, C vs. S)

Maa. Husbandry, migratory, no local headman, occasional polygyny where co-wives occupy separate rooms. (A, B, D, E vs. T, U)

Supyire. Agriculture, bride-price/service, headman a patrilineal heir, high god present but unconcerned with human affairs. (D vs. U, V, W, X, Y)

Afar. Husbandry, clans, seminomadic, hereditary slavery, wealth distinctions, active high gods, insistence on virginity. (C vs. S, V, Y)

Mara Chin. Agriculture, occasional polygyny, aristocracy, hereditary slavery, hereditary headman succession, primogeniture, active high gods. (C vs. R, U, V, X)

Hmong. Agriculture, segmented, bilateral, separated hamlets, no high gods, equally distributed patrilineal inheritance. (C, D vs. V, W, Y)

Enga. Agriculture/pigs, occasional polygyny, no local headman, insistence on virginity. (C, D, E vs. S, Z)

Wik-Mungkan. Hunting/gathering, migratory, very small communities, dowry, no individual land rights. (A, B, C, D, F)

Fijian. Fishing/agriculture/pigs, segmented, no high gods, early marriage age. (C vs. S, W, Y)

Inuktitut. Hunting/fishing, virilocal, bilateral, seminomadic, very small communities, hereditary headman succession, no individual land rights, early marriage age. (A, C, D, F)

Hopi. Agriculture/sheep, matrilocal, matrilineal, no high gods, early marriage age. (C, D vs. W, X, Z)

Kuna. Agriculture/fishing/pigs, bride-service, uxorilocal, demes, bilateral, wealth distinctions, slavery, nonhereditary headman, active high gods, insistence on virginity. (C vs. S, X)

For comparison purposes, ethnography of a contemporary Western community:

New England. Agriculture/husbandry/fishing, neolocal, bilateral, communities of city size, complex social classes, active high gods. (C vs. S, T, V, W, X, Y, Z)

Note. Features from Murdock (1967). Characteristics Service (1971) associated with a more egalitarian band organization: $\mathrm{A}$ - absence of agriculture, $\mathrm{B}$ - migratory or nomadic settlement pattern, $\mathrm{C}$ - no caste stratification, $\mathrm{D}$ - no class stratification, E - no headman/chief, F - very small community size. Characteristics Service associated with a more hierarchical chiefdom (and state) organization: $\mathrm{R}$ - hereditary aristocracy, $\mathrm{S}$ - wealth distinctions, $\mathrm{T}$ - high reliance on animal husbandry, $\mathrm{U}$ - despised occupational groups (incipient caste stratification), $\mathrm{V}$ - some occupational specialization, $\mathrm{W}$ - intensive cultivation or extensive horticulture, $\mathrm{X}$ - compact and relatively permanent settlements, $\mathrm{Y}$ very many levels in jurisdictional hierarchy, $\mathrm{Z}$ - large mean community size. 